\title{
Co-segregation of candidate polymorphism rs201204878 of the PKD1 gene in a large Iranian family with autosomal dominant polycystic disease
}

\author{
FARIBA RANJZAD ${ }^{1}$, AHMAD TARA ${ }^{1}$, ABBAS BASIRI $^{1}$, NASSER AGHDAMI $^{2,3}$ and REZA MOGHADASALI ${ }^{2,3}$ \\ ${ }^{1}$ Urology and Nephrology Research Center, Shahid Beheshti University of Medical Sciences, Tehran-1666677951; \\ Departments of ${ }^{2}$ Stem Cells and Developmental Biology, and ${ }^{3}$ Regenerative Biomedicine, Cell Science Research Center, \\ Royan Institute for Stem Cell Biology and Technology, ACECR, Tehran-8158968433, Islamic Republic of Iran
}

Received February 21, 2017; Accepted September 1, 2018

DOI: $10.3892 /$ etm.2019.7693

\begin{abstract}
Autosomal dominant polycystic kidney disease (ADPKD) is the fourth most common cause of end-stage renal disease, occurring at a frequency of 1 in 400 to 1 in 800 individuals among different populations. The disease affects all ethnic groups worldwide, and there is a requirement for population-based studies to be conducted in order to improve diagnosis, genetic counseling and treatment. A large Iranian family with ADPKD was recruited for the current study. Clinical evaluation was performed to diagnose and assess disease progression in 11 members of this family, including 7 affected members and 4 unaffected members. $P K D 1$ and $P K D 2$ genes were genotyped in subjects by next-generation sequencing (NGS). Mutational analysis of $P K D 1$ and $P K D 2$ genes in this family revealed three intronic variations and three synonymous exonic variants in the $P K D 2$ gene, and two non-synonymous exonic variants and eight intronic variants in $P K D 1$, resulting in a total of 16 heterozygous variations among these two genes. Among the 16 variations, all except three intronic variants in the $P K D 1$ gene have already reported in the Iranian population. The three novel mutations were predicted to be deleterious polymorphisms using in silico methods. Among the reported intronic variations, rs201204878 was identified as a splice region variant, leading to truncation of the polycystin-1 protein. In conclusion, genotyping of $P K D 1$ and $P K D 2$ in this family with ADPKD revealed no mutational hot spots. However, genetic screening identified three novel variants in the Iranian population. The data generated in the present study
\end{abstract}

Correspondence to: Dr Abbas Basiri, Urology and Nephrology Research Center, Shahid Beheshti University of Medical Sciences, 103 9th Boustan Street, Pasdaran Ave., Tehran-1666677951, Islamic Republic of Iran

E-mail: basiri@unrc.ir

Key words: polycystic kidney disease, autosomal dominant polycystic kidney disease, $P K D 1, P K D 2$, end-stage renal disease, next-generation sequencing, novel variant will contribute to improving the diagnosis, genetic counseling and treatment of patients with ADPKD.

\section{Introduction}

Autosomal dominant polycystic kidney disease (ADPKD) is the most common hereditary kidney disorder found among all ethnic groups worldwide, affecting 1 in 400 to 1 in 800 live births among different populations $(1,2)$. The disease is characterized by the development and progressive enlargement of renal cysts and is the fourth most common cause leading to end-stage renal disease (ESRD) $(2,3)$. The etiology of this disease is associated with mutations in PKD1 [Online Mendelian Inheritance in Man (OMIM) no. 601313; 16p13.3] and PKD2 (OMIM no. 173910; 4q21-22) genes, and 85-90\% of ADPKD cases are caused primarily by mutations in the $P K D 1$ gene (4). $P K D 1$ has 46 exons and encodes the transmembrane protein, polycystin-1 (PC1), which is composed of 4,303 amino acids. PKD2 is a smaller gene with 15 exons. It encodes the transmembrane protein polycystin-2 (PC2), which is composed of 968 amino acids $(5,6)$. PC1 contains an $\mathrm{N}$-terminal extracellular region, 11 membrane-spanning domains and a cytoplasmic C-tail, whereas the PC2 protein possesses a shorter N-terminal extracellular region and only 6 membrane-spanning domains $(7,8)$.

The diagnosis of ADPKD is a challenge; renal ultrasound, magnetic resonance imaging and computed tomography have limited capability in terms of detecting ADPKD in patients (9). With no hotspot mutation information available for PKD1 and PKD2, clinical molecular diagnostic techniques are difficult (10). To overcome these challenges, the genotype variation of these two genes in patients with ADPKD has been investigated using techniques including polymerase chain reaction (PCR)-single-strand conformation polymorphism, denaturing high-performance liquid chromatography, multiplex ligation-dependent probe amplification and next-generation sequencing (NGS) (11-14). Recent advancements in sequencing technologies have enabled the rapid and cost-effective generation of large quantities of data. By removing most of the throughput and resource limitations of traditional methods, NGS enables investigators to analyze $P K D 1$ and $P K D 2$ genes in a single run (15-17). 
In individuals diagnosed with ADPKD, PKDI exhibits marked allelic heterogeneity, with a high level of gene variation $(18,19)$. To date, $>2,300$ germline mutations of $P K D 1$ have been identified; $~ 900$ are likely to be neutral polymorphisms and $>150$ are unclassified variants (20). In the current study, the pathogenicity of an atypical splice variant in the $P K D 1$ gene, rs201204878, was evaluated in an Iranian family with ADPKD. In silico prediction-based models were used to evaluate its pathogenicity.

\section{Materials and methods}

Ethical compliance. The study was approved by the ethics committee board of Shahid Beheshti University of Medical Sciences (Tehran, Iran) and all experiments were performed in adherence to the declaration of Helsinki. Written informed consent was obtained from all patients prior to their participation in the study.

Patient pedigree and subject data. A large Iranian family of 19 members ( 10 males, nine females; age, $40.8 \pm 15.7$ years) with a history of ADPKD was recruited for this study from dialysis centers and nephrology clinics of Modarres Hospital (Tehran, Iran) in June 2015. In the pedigree, 11 members were recruited; seven of them were diagnosed with ADPKD by ultrasound examination, according to Ravine's criteria (21). The proband selected was diagnosed with ESRD, and had two affected brothers and two affected sisters with early onset ESRD (age of onset, $<50$ years). A screening ultrasound was performed on 11 available participants of the asymptomatic at-risk adult members in this family. Details of the affected and non-affected members of the family (Four generations, 19 members in total) are shown in Fig. 1. Total genomic DNA from 11 available family members was extracted from blood samples using a standard phenol-chloroform procedure as previously described (22). The quality and concentration of DNA samples was evaluated using spectrophotometry $(260 / 280 \mathrm{~nm})$ and $1 \%$ agarose gel electrophoresis.

Mutational analysis by targeted NGS. Targeted NGS experiments were performed by BGI Tech Europe (Copenhagen, Denmark). The Genetic Sequencing Test was performed using a custom-designed NimbleGen chip (Roche NimbleGen, Inc., Madison, WI, USA) by BGI Tech Europe to capture the genes of interest, which were then sequenced by NGS. In general, the test platform examined $>95 \%$ of the target gene with a sensitivity of $>99 \%$. Point mutations, micro-insertions, deletions and duplications $(<20 \mathrm{bp})$ were detected simultaneously (14). SNPs and indels were identified via the SOAP snp software (Release 1.03; http://soap.genomics.org. cn/soapsnp.html) and GATK Indel Genotyper (Version 4.0.11; broadinstitute.org/gsa/wiki/index.php/).

Bioinformatics analysis and mutation identification. Candidate variations were compared against electronic database information and computational analysis was performed. The detected sequence variations were compared against the currently published list of PKD gene variants in the Human Gene Mutation Database (23), the Autosomal Dominant Polycystic Kidney Disease Mutation Database version 3.1 (20) and the Iranian Human Mutation Gene Bank (24). The effects of variation were analyzed using web-based computational

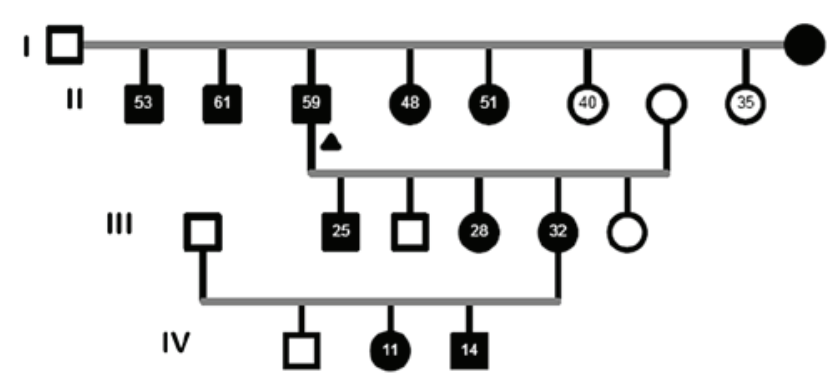

Figure 1. Pedigree of an Iranian family with autosomal dominant polycystic kidney disease. The proband is indicated with an arrowhead. The shape described the sex (circles were identified as female and square were identified as male), the number represents the age and the solid black shapes represent the individuals recruited for the present study.

pathogenicity prediction tools, including MutationTaster working on current build of NCBI 137/Ensembl 69 (25), Sorting Intolerant from Tolerant based on NCBI 137/Ensembl 66 (26) and Polymorphism Phenotyping version 2 (27). Human Splice Finder (HSF) software version 3.0 was also used to predict splicing (28).

Sanger sequencing. To validate the variants predicted to be associated with ADPKD by computational analyses, the region of interest surrounding the variant was amplified by PCR using Taq DNA Polymerase Master Mix (Ampliqon A/S, Odense, Denmark). The variants were sequenced using Sanger sequencing methods and appropriate internal primers reported previously (Table I) (29).

Segregation analysis. To confirm the association between mutation and the pathogenicity of the disease, analysis of seven affected family members in the pedigree was performed by direct sequencing (Sanger method as previously described). The Iranian normal population database contains data of the reported variants. Four unaffected family members and four normal controls from the Iranian normal population database were also checked for the mutation to confirm the prediction.

\section{Results}

Mutational analysis. Mutational analysis of the $P K D 1$ and $P K D 2$ genes was performed in a large Iranian family consisting of 11 members (5 male and 6 females); 7 of them (3 male and 4 female) diagnosed with ADPKD by renal ultrasound. Using targeted NGS, three intronic variations and three synonymous exonic variants were identified in the PKD2 gene, and two non-synonymous exonic variants and eight intronic variants were identified in the PKDl gene (Table II). All variants except from three intronic variants in $P K D 1$ have been reported in the National Centre for Biotechnology Information dbSNP database (build 151; ncbi.nlm.nih.gov/SNP/) and are considered to be known polymorphisms. The three novel mutations were predicted to be deleterious polymorphisms by bioinformatics analysis.

Analysis of splice site mutations. Among the three reported non-coding RNA (ncRNA) intronic variations, one (rs201204878) was identified as a splice region variant (c. $11537+5 \_6$ ins ccc). The pathogenic relevance of putative splice site mutations was 
Table I. Primers employed for Sanger sequencing.

\begin{tabular}{llccr}
\hline Exon & \multicolumn{1}{c}{ Primer sequence $\left(5^{\prime}-3^{\prime}\right)$} & PCR annealing temperature $\left({ }^{\circ} \mathrm{C}\right)$ & Cycle & (Refs.) \\
\hline 41 & F-CGGCCTCCTGACCAGCCTGGCTC & 64 & 30 & $(31)$ \\
& R-TAGGCCAGCGGGGGCCGGAGGAGTG & & & \\
\hline
\end{tabular}

PCR, polymerase chain reaction; F, forward; R, reverse.

Table II. Mutations identified in $P K D 1$ and $P K D 2$ genes in a large Iranian family with autosomal dominant polycystic kidney disease.

\begin{tabular}{|c|c|c|c|c|c|}
\hline Gene & Genomic position & RefSeq & Nucleic acid alteration & Mutation location & SNPID \\
\hline PKD2 & $88,957,562$ & NM_000297 & $\mathrm{T} / \mathrm{C}$ & Intronic & rs17786456 \\
\hline PKD2 & $88,959,381$ & NM_000297 & $\mathrm{G} / \mathrm{A}$ & Intronic & rs 2725221 \\
\hline PKD2 & $88,959,745$ & NM_000297 & $\mathrm{A} / \mathrm{G}$ & Intronic & rs17013735 \\
\hline PKD1 & $2,140,294$ & NM_001009944 & $\mathrm{C} / \mathrm{T}$ & Exonic & rs148478410 \\
\hline PKD1 & $2,141,714$ & NM_001009944 & $\mathrm{G} / \mathrm{A}$ & ncRNA_intronic & Novel \\
\hline PKD1 & $2,141,776$ & NM_001009944 & $-/ \mathrm{CCC}$ & ncRNA_intronic & rs201204878 \\
\hline PKD1 & $2,142,113$ & NM_001009944 & $\mathrm{G} / \mathrm{A}$ & Exonic & rs145955373 \\
\hline PKD1 & $2,152,574$ & NM_001009944 & $\mathrm{C} / \mathrm{T}$ & Exonic & rs374619113 \\
\hline$P K D 1$ & $2,154,478$ & NM_001009944 & $\mathrm{A} / \mathrm{G}$ & Intronic & rs4786209 \\
\hline PKD1 & $2,156,369$ & NM_001009944 & $\mathrm{G} / \mathrm{A}$ & Intronic & rs 142761413 \\
\hline PKD1 & $2,158,176$ & NM_001009944 & $\mathrm{T} / \mathrm{A}$ & Intronic & rs200363107 \\
\hline PKD1 & $2,163,115$ & NM_001009944 & $\mathrm{G} / \mathrm{C}$ & ncRNA_intronic & Novel \\
\hline PKD1 & $2,163,579$ & NM_001009944 & $\mathrm{G} / \mathrm{C}$ & Intronic & rs56326527 \\
\hline PKDI & $2,164,808$ & NM_001009944 & $\mathrm{C} / \mathrm{T}$ & Exonic & rs40433 \\
\hline PKD1 & $2,166,769$ & NM_001009944 & $\mathrm{G} / \mathrm{A}$ & Intronic & Novel \\
\hline PKDI & $2,167,874$ & NM_001009944 & $\mathrm{G} / \mathrm{A}$ & Exonic & rs 35842 \\
\hline
\end{tabular}

SNP, single nucleotide polymorphism; ncRNA, non-coding RNA.

evaluated using HSF software. The HSF results indicated that this mutation occurred in the late exonic positions and generated a new exonic splice site that potentially altered splicing. Therefore this mutation was classified as a highly likely pathogenic mutation (30). This variant was assessed for its co-segregation with the disease in three affected and two unaffected family members The mutation was identified in all affected family members, and was absent in unaffected family members, as well as in 400 normal subjects in the Iranian population (Fig. 2).

Segregation analysis. In the present study, the allele frequency of the c. $11537+5 \_6$ ins ccc variation in world population groups from the 1,000Genomes Project was analyzed using the Ensembl database (internationalgenome.org/category/ensembl/). The frequency of this mutation in sub-populations from America, Africa and East Asia was 0\%. The frequency of this mutation in European and South Asian sub-populations was 2 and $3 \%$, respectively. However, the homozygous genotype frequency in these sub-populations was $0 \%$. This appears to be consistent with the experimental results of the current study, and may be associated with the truncation effect of this variant and the subsequent abortion of embryos carrying the homozygous mutation in mice models (31). The co-segregation analysis and
HSF of rs201204878 showed no co-relation with the severity of ADPKD in the affected family members.

\section{Discussion}

According to the genotype-phenotype correlation, the ADPKD phenotype exhibits both genetic and allelic dependency. PKDI mutations are correlated with disease severity and the onset of ESRD in ADPKD patients (32). The c. $11537+5$ _6 ins ccc splice mutation identified in the present study is a type of truncating mutation, which has been associated with a more severe ADPKD phenotype when compared with non-truncating PDK1 mutations (4). To the best of our knowledge, the present study is the first to report the rs201204878 pathogenic variant in the Iranian population, as this variation was not observed in 400 subjects from the Iranian normal population database. The clinical severity of ADPKD in affected family members was consistent with the obtained splicing mutation results from HSF and co-segregation analyses. Hence, follow-up in other young affected family members becomes necessary to verify the reported association. The average age of ESDR onset varies with the type of gene involved, and it was reported to be 54.3 years in patients with PKD1 mutations and 74 years in patients with PKD2 mutations (33). 

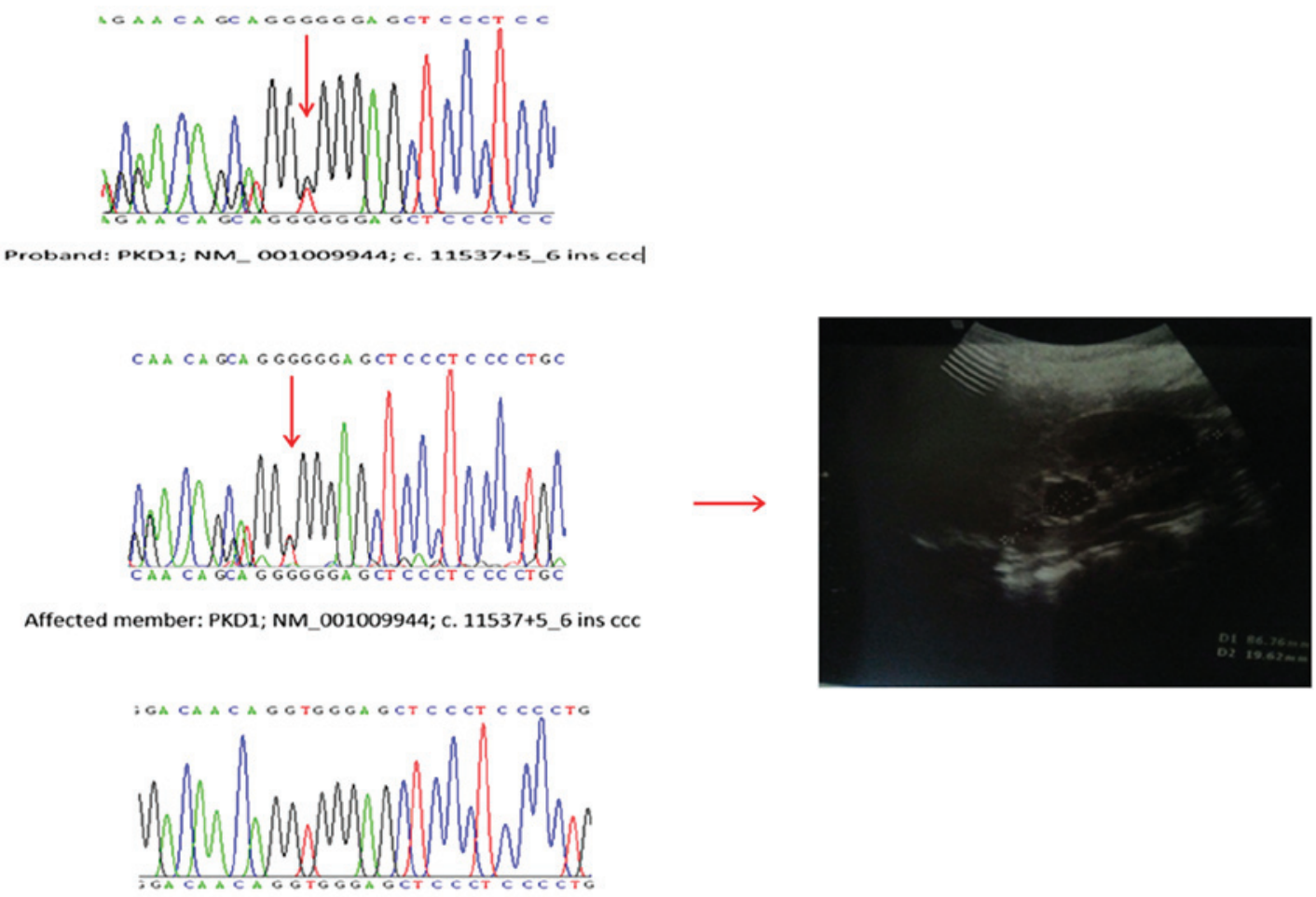

Normal member: PKD1; NM_001009944

Figure 2. Identification of the heterozygote mutation. The heterozygote mutation c. 11537+5_6 ins ccc on PKD1 gene was identified by DNA sequencing in the antisense strands of proband and affected family members, but was not identified in unaffected family members. The ultrasound examination of an affected family member is demonstrated on the left.

In the present study, the heterozygous $11537+5$ _6 ins ccc mutation (rs201204878) was identified in intron 41 of the PKDl gene, and no other truncation mutations were identified by targeted NGS. This was demonstrated to be a pathogenic mutation, which extends the associated phenotypic and genotypic spectrums for ADPKD. The rs201204878 mutation has been previously reported to be benign (33), but in the present study it was predicted that the splice mutation may lead to abnormal splicing of PKD1. This is expected to affect $\mathrm{PC} 1$ function, which is consistent with a previous report demonstrating that the expression of the truncated protein is correlated with the early onset of ESRD (34).

In the current study, targeted NGS of the PKD1 and PKD2 genes produced high-coverage sequencing data with high sensitivity and specificity. The results were confirmed with Sanger sequencing, which is the standard approach for clinical genetic testing in ADPKD. The results suggest that targeted NGS may potentially replace Sanger sequencing for clinical genetic testing in ADPKD as the former is a faster and more accurate procedure In silico and co-segregation analyses concluded that the rs201204878 variant may be considered as a potential functional mutation. However, further mini gene analysis may provide further insight into the pathogenicity of this mutation. In addition, as the Iranian population is not included in the 1,000 Genomes Project (35), performing genetic variation studies will add to the plethora of data available on mutations; such studies on ethnic populations may aid in the diagnosis, prognosis and management of this disease. In conclusion, the polymorphisms identified in the present study may contribute to improving the diagnosis, genetic counseling and treatment of patients with ADPKD.

\section{Acknowledgements}

The authors want to thank the Urology and Nephrology Research Center (UNRC) of Shahid Beheshti University of Medical Sciences for their support.

\section{Funding}

This trial was supported by a grant from Royan Institute and the Royan Charity Association for Health Research (grant no. 93/566).

\section{Availability of data and materials}

The datasets used and/or analyzed during the current study are available from the corresponding author on reasonable request.

\section{Authors' contributions}

$\mathrm{AB}$ conceived of the present study. FR and AT performed the experiments. AB, NA and RM analysed the results. FR and $\mathrm{AB}$ wrote the manuscript. NA and RM edited the manuscript.

\section{Ethics approval and consent to participate}

The study was approved by the ethics committee board of Shahid Beheshti University of Medical Sciences (Tehran, Iran) and all experiments were performed in adherence to the declaration of Helsinki. Written informed consent was obtained from all patients prior to their participation in the study. 


\section{Consent for publication}

Not applicable.

\section{Competing interests}

The authors declare that they have no competing interests.

\section{References}

1. Trujillano D, Bullich G, Ossowski S, Ballarín J, Torra R, Estivill X and Ars E: Diagnosis of autosomal dominant polycystic kidney disease using efficient PKD1 and PKD2 targeted next-generation sequencing. Mol Genet Genomic Med 2: 412-421, 2014.

2. Wilson PD: Polycystic kidney disease. N Engl J Med 350: 151-164, 2004.

3. Srivastava A and Patel N: Autosomal dominant polycystic kidney disease. Am Fam Physician 90: 303-307, 2014.

4. Kurashige M, Hanaoka K, Imamura M, Udagawa T, Kawaguchi Y, Hasegawa T, Hosoya T, Yokoo T and Maeda S: A comprehensive search for mutations in the PKD1 and PKD2 in Japanese subjects with autosomal dominant polycystic kidney disease. Clin Genet 87: 266-272, 2015.

5. Germino GG, Weinstat-Saslow D, Himmelbauer H, Gillespie GA Somlo S, Wirth B, Barton N, Harris KL, Frischauf AM and Reeders ST: The gene for autosomal dominant polycystic kidney disease lies in a 750-kb CpG-rich region. Genomics 13: 144-151, 1992.

6. Cornec-Le Gall E, Audrézet MP, Le Meur Y, Chen JM and Férec C: Genetics and pathogenesis of autosomal dominant polycystic kidney disease: 20 years on. Hum Mutat 35: 1393-1406, 2014.

7. Hughes J, Ward CJ, Peral B, Aspinwall R, Clark K, San Millán JL, Gamble V and Harris PC: The polycystic kidney disease 1 (PKD1) gene encodes a novel protein with multiple cel recognition domains. Nat Genet 10: 151-160, 1995.

8. Nims N, Vassmer D and Maser RL: Transmembrane domain analysis of polycystin-1, the product of the polycystic kidney disease-1 (PKD1) gene: Evidence for 11 membrane-spanning domains. Biochemistry 42: 13035-13048, 2003.

9. Pei Y, Obaji J, Dupuis A, Paterson AD, Magistroni R, Dicks E, Parfrey P, Cramer B, Coto E, Torra R, et al: Unified criteria for ultrasonographic diagnosis of ADPKD. J Am Soc Nephrol 20: 205-212, 2009.

10. Tan AY, Michaeel A, Liu G, Elemento O, Blumenfeld J, Donahue S, Parker T, Levine D and Rennert H: Molecular diagnosis of autosomal dominant polycystic kidney disease using next-generation sequencing. J Mol Diagn 16: 216-228, 2014.

11. Ding L, Zhang S, Qiu W, Xiao C, Wu S, Zhang G, Cheng L and Zhang S: Novel mutations of PKD1 gene in Chinese patients with autosomal dominant polycystic kidney disease. Nephrol Dial Transplant 17: 75-80, 2002

12. Yu C, Yang Y, Zou L, Hu Z, Li J, Liu Y, Ma Y, Ma M, Su D and Zhang S: Identification of novel mutations in Chinese Hans with autosomal dominant polycystic kidney disease. BMC Med Genet 12: 164, 2011

13. Liu G, Tan AY, Michaeel A, Blumenfeld J, Donahue S, Bobb W, Parker T, Levine D and Rennert H: Development and validation of a whole genome amplification long-range PCR sequencing method for ADPKD genotyping of low-level DNA samples. Gene 550: 131-135, 2014

14. Yang T, Meng Y, Wei X, Shen J, Zhang M, Qi C, Wang C, Liu J, $\mathrm{Ma} M$ and Huang S: Identification of novel mutations of PKD1 gene in Chinese patients with autosomal dominant polycystic kidney disease by targeted next-generation sequencing. Clin Chim Acta 433: 12-19, 2014.

15. Rossetti S, Hopp K, Sikkink RA, Sundsbak JL, Lee YK, Kubly V, Eckloff BW, Ward CJ, Winearls CG, Torres VE and Harris PC: Identification of gene mutations in autosomal dominant polycystic kidney disease through targeted resequencing. J Am Soc Nephrol 23: 915-933, 2012.

16. Eisenberger T, Decker C, Hiersche M, Hamann RC, Decker E, Neuber S, Frank V, Bolz HJ, Fehrenbach H, Pape L, et al: An efficient and comprehensive strategy for genetic diagnostics of polycystic kidney disease. PloS One 10: e0116680, 2015.
17. Edrees BM, Athar M, Al-Allaf FA, Taher MM, Khan W, Bouazzaoui A, Al-Harbi N, Safar R, Al-Edressi H, Alansary K, et al: Next-generation sequencing for molecular diagnosis of autosomal recessive polycystic kidney disease. Gene 591: 214-226, 2016.

18. Harris PC and Hopp K: The mutation, a key determinant of phenotype in ADPKD. J Am Soc Nephrol 24: 868-870, 2013

19. Li J, Yu C, Tao Y, Yang Y, Hu Z and Zhang S: Putative mutation of PKD1 gene responsible for autosomal dominant polycystic kidney disease in a Chinese family. Int J Urol 18: 240-242, 2011

20. Gout AM, Martin NC, Brown AF and Ravine D: PKDB: Polycystic kidney disease mutation database-a gene variant database for autosomal dominant polycystic kidney disease. Hum Mutat 28: 654-659, 2007.

21. Ravine D, Gibson RN, Walker RG, Sheffield LJ, Kincaid-Smith P and Danks DM: Evaluation of ultrasonographic diagnostic criteria for autosomal dominant polycystic kidney disease 1. Lancet 343: 824-827, 1994

22. Sambrook J and Russell DW: Purification of nucleic acids by extraction with phenol: Chloroform. CSH Protoc 2006: pdb. prot4455, 2006.

23. Stenson PD, Mort M, Ball EV, Howells K, Phillips AD, Thomas NS and Cooper DN: The human gene mutation database: 2008 update. Genome Med 1: 13, 2009.

24. Schwarz JM, Rödelsperger C, Schuelke M and Seelow D: MutationTaster evaluates disease-causing potential of sequence alterations. Nat Methods 7: 575-576, 2010.

25. Kumar P, Henikoff S and Ng PC: Predicting the effects of coding non-synonymous variants on protein function using the SIFT algorithm. Nat Protoc 4: 1073-1081, 2009.

26. Adzhubei IA, Schmidt S, Peshkin L, Ramensky VE, Gerasimova A, Bork P, Kondrashov AS and Sunyaev SR: A method and server for predicting damaging missense mutations. Nat Methods 7: 248-249, 2010

27. Desmet FO, Hamroun D, Lalande M, Collod-Béroud G, Claustres M and Béroud C: Human Splicing Finder: An online bioinformatics tool to predict splicing signals. Nucleic Acids Res 37: e67, 2009.

28. Rossetti S, Chauveau D, Walker D, Saggar-Malik A, Winearls CG, Torres VE and Harris PC: A complete mutation screen of the ADPKD genes by DHPLC. Kidney Int 61: $1588-1599,2002$

29. Kinoshita M, Higashihara E, Kawano H, Higashiyama R, Koga D, Fukui T, Gondo N, Oka T, Kawahara K, Rigo K, et al: Technical evaluation: Identification of pathogenic mutations in PKD1 and PKD2 in patients with autosomal dominant polycystic kidney disease by next-generation sequencing and use of a comprehensive new classification system. PloS One 11: $\mathrm{e} 0166288,2016$.

30. Flaherty L, Bryda EC, Collins D, Rudofsky U and Montgomery JC: New mouse model for polycystic kidney disease with both recessive and dominant gene effects. Kidney Int 47: 552-558, 1995.

31. Cornec-Le Gall E, Audrézet MP, Chen JM, Hourmant M, Morin MP, Perrichot R, Charasse C, Whebe B, Renaudineau E, Jousset P, et al: Type of PKD1 mutation influences renal outcome in ADPKD. J Am Soc Nephrol 24: 1006-1013, 2013.

32. Heyer CM, Sundsbak JL, Abebe KZ, Chapman AB, Torres VE, Grantham JJ, Bae KT, Schrier RW, Perrone RD, Braun WE, et al: Predicted mutation strength of nontruncating PKD1 mutations aids genotype-phenotype correlations in autosomal dominant polycystic kidney disease. J Am Soc Nephrol 27: 2872-2884, 2016.

33. Sherry ST, Ward MH, Kholodov M, Baker J, Phan L, Smigielski EM and Sirotkin K: dbSNP: The NCBI database of genetic variation. Nucleic Acids Res 29: 308-311, 2001.

34. Gainullin VG, Hopp K, Ward CJ, Hommerding CJ and Harris PC: Polycystin-1 maturation requires polycystin-2 in a dose-dependent manner. J Clin Invest 125: 607-620, 2015.

35. Siva N: 1000 Genomes project. Nat Biotechnol 26: 256, 2008. 